\title{
Problemas Relacionados aos Medicamentos em pacientes atendidos no pronto socorro de um Hospital Universitário
}

\author{
Drug-Related Problems in Emergency Room Patients at a \\ University Hospital
}

Recebido em: 27/04/2020 Aceito em: $25 / 08 / 2020$
Paulimira Fernanda da CRUZ1 ${ }^{1}$; Amanda de Cássia PAIVA ${ }^{1}$; Miguel Simião PEREIRA JÚNIOR ${ }^{2}$; Jaqueline Jóice MUNIZ ${ }^{1}$

${ }^{1}$ Universidade do Vale do Sapucaí (UNIVAS). Avenida Cel. Alfredo Custódio de Paula, 320, CEP 37553-068. Pouso Alegre, MG, Brasil. ${ }^{2}$ Hospital das Clinicas Samuel Libânio (HCSL). Avenida Cel. Alfredo Custódio de Paula, 320, CEP 37553-068. Pouso Alegre, MG, Brasil.

E-mail: jaquelinejoice@yahoo.com.br

\section{ABSTRACT}

Drug-related problems (DRP) are caused by failures that affect the effectiveness and safety of drugs and represent an important challenge for health systems. This study identified and evaluated cases of MRP in the Emergency Room (ER) of Hospital das Clínicas Samuel Libânio (HCSL).It is a descriptive and cross-sectional study conducted through interviews with patients who sought care at the HCSL ER. The identification and classification of the DRP was selected based in the Second Consensus of Granada. Of the 100 patients interviewed, there was a high prevalence of DRPs in the group of patients over 60 years old (46\%), but there was no significant difference between female (24\%) and male (22\%). Most respondents had little adherence to treatment (68\%) and low education level, with no elementary education $(62 \%)$. Among all types of DRP, the most prevalent were quantitative ineffectiveness (78\%) and untreated health problem (16\%). The DRP cases at HCSL ER are common in patients with chronic diseases aged over 60 years due to non-adherence to treatment. These results demonstrate the importance of implementing pharmacotherapeutic monitoring based on education and primary health care.

Keywords: pharmacotherapy; drug-related problems; emergency room; adverse drug reactions; use of medicine.

\section{RESUMO}

Problemas relacionados aos medicamentos (PRM) são causados por falhas que afetam a efetividade e segurança dos medicamentos e representam um importante desafio para os sistemas de saúde. Este estudo teve por objetivo identificar e avaliar casos de PRM no Pronto Socorro (PS) do Hospital das Clínicas Samuel Libânio (HCSL). Trata-se de um estudo descritivo e transversal, realizado por meio de entrevistas a pacientes que procuraram atendimento no PS do HCSL. A identificação e classificação dos PRM foram baseadas no Segundo Consenso de Granada. Dos 100 pacientes entrevistados, houve alta prevalência de PRM no grupo de pacientes acima de 60 anos (46\%), porém não houve diferença significativa entre sexo feminino (24\%) e 
masculino (22\%). Grande parte dos entrevistados teve pouca adesão ao tratamento (68\%) e nível de escolaridade baixo, sem ensino fundamental (62\%). Entre os avaliados, todos apresentaram algum tipo de PRM, sendo os mais encontrados: inefetividade quantitativa (78\%) e problema de saúde não tratado (16\%). Os casos de PRM atendidos no PS do HCSL são comuns em pacientes com doenças crônicas, com idade acima de 60 anos, devido a não adesão ao tratamento. Esses resultados demonstram a importância da implantação do seguimento farmacoterapêutico pautado na educação e atenção primária à saúde.

Palavras-chave: farmacoterapia; problemas relacionados com medicamentos; pronto-socorro; reações adversas a medicamentos; utilização de medicamentos.

\section{INTRODUÇÃO}

O consumo abusivo de medicamentos, associado ao uso inadequado e a utilização desses sem prescrição médica ou orientação farmacêutica, é um grave problema de saúde pública no Brasil (1). Dados sobre a utilização incorreta e insegura de medicamentos no Brasil são alarmantes. No país, a automedicação é uma prática comum; existe má qualidade de oferta de medicamentos, não se cumpre a obrigatoriedade da receita médica, além da carência de informação e instrução da população em geral, justificando assim a preocupação com a implementação de estratégias para evitar o uso irracional de medicamentos (2).

Apesar de existir estratégias para promoção do uso racional de medicamentos, estudos ainda apontam a presença de problemas de saúde cuja origem está relacionada ao uso incorreto de medicamentos (3). As falhas terapêuticas ocorrem quando os medicamentos causam danos, não são seguros (intoxicações, reações adversas), e/ou quando não apresentam ou deixam de apresentar eficácia desejada, não atingindo o objetivo para o qual foram prescritos (4).

Problemas relacionados aos medicamentos (PRM) são definidos como qualquer situação em que, no processo de uso de medicamentos, resulte em um resultado negativo associado ao medicamento (RNM) (5). Os RNM são alterações não desejadas no estado de saúde do paciente causados pela farmacoterapia, são produzidos por diversas causas e têm como consequência o não alcance do objetivo terapêutico desejado ou surgimento de efeitos não desejados (6).
A morbimortalidade relacionada aos medicamentos advém dos PRM, os quais afetam grande parcela da população que utilizam medicamentos de forma incorreta. Diversas publicações têm mostrado que os PRM, além de influenciar negativamente na evolução clínica dos pacientes, são responsáveis por um número considerável de hospitalizações e morte a cada ano, gerando um aumento significativo dos custos para os sistemas de saúde (7).

As principais causas dessas morbimortalidades são as prescrições inadequadas, reações adversas aos medicamentos (RAM), não adesão ao tratamento, superdosagem ou subdosagem, não cumprimento do regime terapêutico (utilização incorreta dos medicamentos), inadequado seguimento de sinais e sintomas, além da saúde da população (7).

Um estudo realizado no sul do Brasil, por Dall' Agnol (2003), demonstrou que um terço dos pacientes que procuraram atendimento na emergência do hospital, o fizeram devido a PRM (3). Nesse contexto, no Brasil não há muitos estudos acerca de identificação e caracterização de PRM. Além disso, pesquisas que indicam o impacto econômico de PRM para os sistemas de saúde são ainda mais incomuns. Diante desse cenário, o propósito deste estudo foi identificar os casos de procura de atendimento no PS do HCSL por PRM e avaliar os tipos mais comumente encontrados.

\section{MÉTODO}

O estudo foi submetido e aprovado pelo Comitê de Ética em pesquisa da Universidade do Vale do Sapucaí (UNIVAS), com número de parecer 2.953.196. Trata-se de estudo descritivo e transver- 
sal realizado no Pronto socorro (PS) do Hospital das Clínicas Samuel Libânio (HCSL) de Pouso Alegre, MG, Brasil, durante o período de janeiro a abril de 2019. O HCSL é um hospital universitário, filantrópico, de alta complexidade e referência nos atendimentos de urgência e emergência, com estimativa de atendimento mensal de aproximadamente 6.000 pacientes.

Todos os pacientes que procuraram atendimento médico no PS, no período de realização do presente estudo, foram entrevistados durante o processo de triagem realizado por enfermeiros. Na entrevista, utilizando formulário adaptado para obtenção do histórico farmacoterapêutico dos pacientes, foram selecionados para participar do estudo aqueles classificados como casos suspeitos de PRM. Foram coletadas as informações sobre os problemas de saúde do paciente, medicamentos em uso, queixas relatadas durante $\mathrm{o}$ atendimento e cor de pulseira recebida. Essa sinalizava o nível de gravidade e a prioridade de atendimento destes pacientes.

Foram incluídos no estudo 100 pacientes que aceitaram participar da pesquisa e assinar o Termo de Consentimento Livre e Esclarecido (TCLE). Os critérios de inclusão foram: indivíduos com idade igual ou superior a 18 anos, de ambos os sexos, capazes de se comunicar ou que estavam acompanhados por cuidador/familiar. Os critérios de exclusão foram: pacientes incapazes de se comunicar e que não estavam acompanhados por cuidador/familiar; pacientes que não aceitaram responder todas as perguntas do formulário; pacientes que desistiram da consulta médica e de assinar o TCLE. Os participantes foram entrevistados após o processo de triagem (antes da consulta médica). Para a entrevista, foi utilizado um instrumento de coleta de dados (formulário) validado em um estudo previamente realizado no hospital privado geral no município de Araraquara, SP, Brasil, em 2016 (8). Para a realização do presente estudo adequações foram feitas e as perguntas foram direcionadas para obtenção do histórico farmacoterapêutico (medicamentos utilizados, posologia prescrita, modo de utilização, hábitos de vida, histórico clínico, dados sociodemográficos dos pacientes).

A identificação e a classificação dos PRM foram baseadas no Segundo Consenso de Granada (6). Os dados obtidos foram tabulados em uma planilha do programa Microsoft Excel 2013 para análise dos resultados.

\section{RESULTADO E DISCUSSÃO}

Dos 100 pacientes avaliados e identificados com PRM, 55\% eram do sexo feminino e $45 \%$ do sexo masculino. Os PRM encontrados foram predominantes na faixa etária acima de 60 anos (totalizando 46\%), porém não mostraram ser diferentes de acordo com o sexo (Figura 1). Estudo anterior já havia mostrado o grande número de casos de PRM em pacientes acima de 65 anos, o que se deve principalmente ao número elevado de medicamentos

Figura 1. Distribuição percentual de idade e sexo de pacientes com Problemas Relacionados aos Medicamentos (PRM) atendidos no pronto socorro do Hospital das Clínicas Samuel Libânio, Pouso Alegre, MG, Brasil (janeiro-abril de 2019).

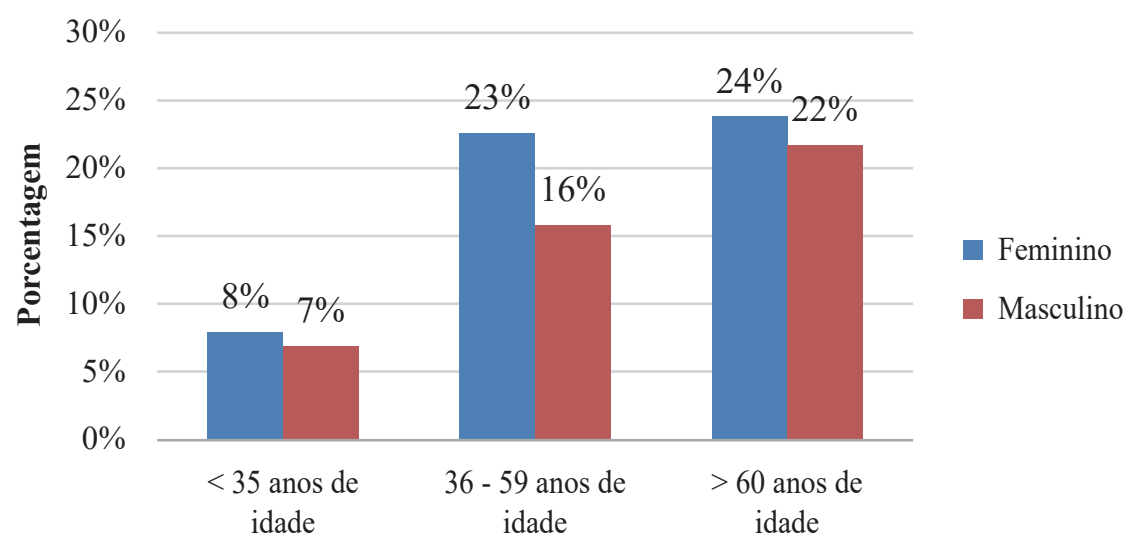


Figura 2. Distribuição percentual de níveis de escolaridade dos pacientes atendidos no pronto socorro do Hospital das Clínicas Samuel Libânio, Pouso Alegre, MG, Brasil (janeiro-abril de 2019).

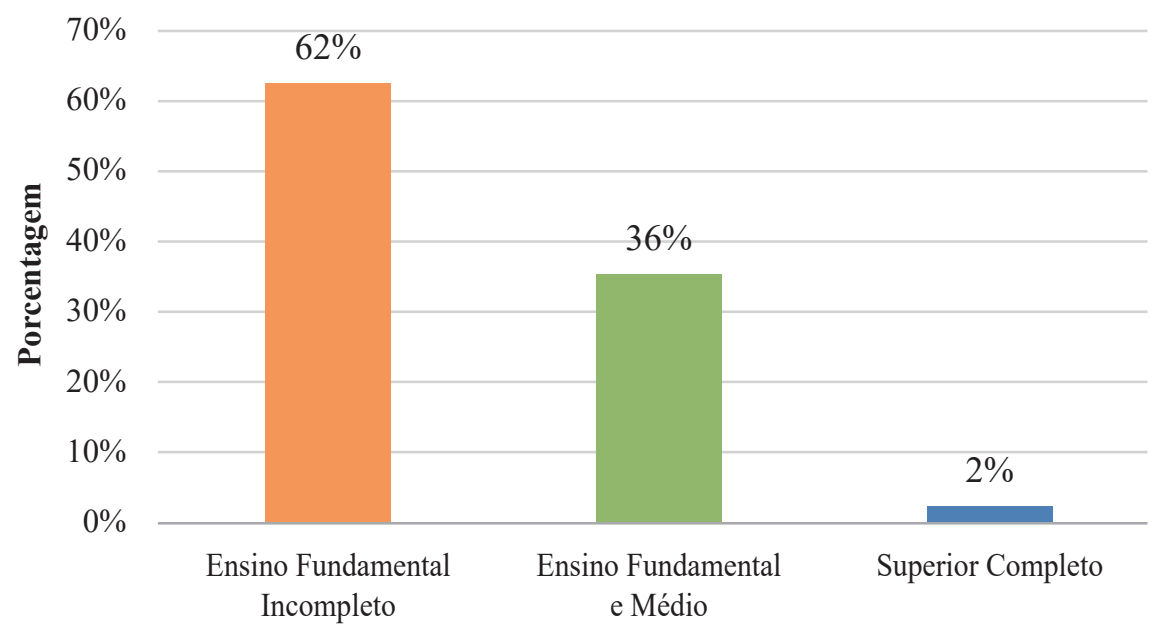

Figura 3. Distribuição percentual de pacientes atendidos no Hospital das Clínicas Samuel Libânio, Pouso Alegre, MG, Brasil, com suspeita de Problemas Relacionados aos Medicamentos (PRM), em relação à cor de pulseiras utilizadas no pronto socorro (janeiro-abril de 2019).

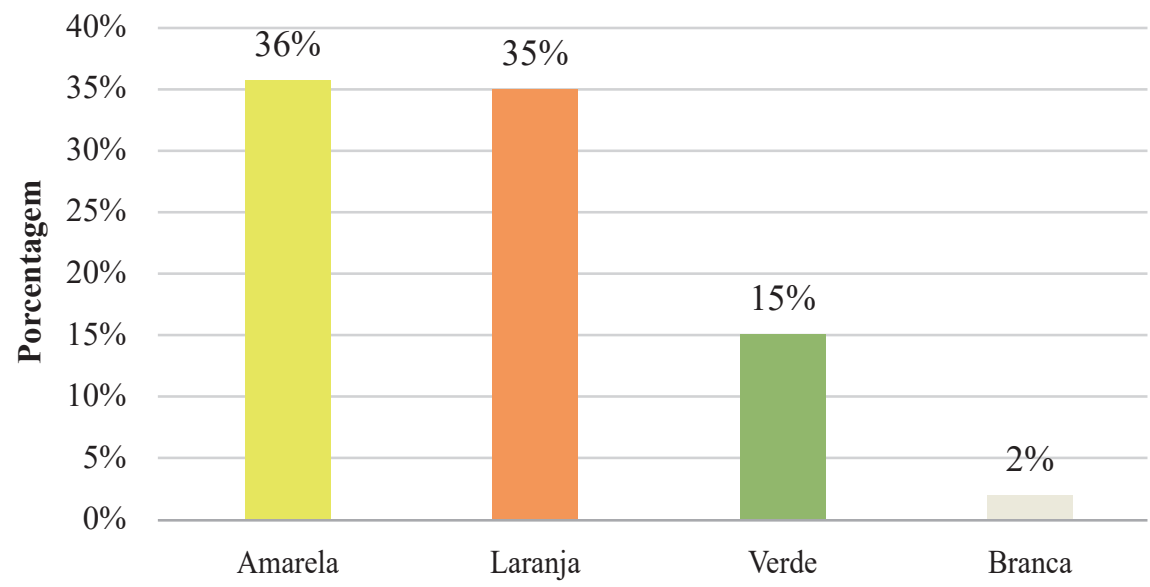

que esse grupo de pacientes utiliza (3). Esses resultados corroboram outras publicações científicas, em que foram observados que as mulheres apresentaram mais PRM do que os homens, com incidência de $50 \%, 65,7 \%$ e $63,6 \%$ (9-12). Acredita-se que a maior procura por serviços de saúde, por mulheres, possa influenciar o maior casos de PRM nesse grupo.

O nível de escolaridade dos pacientes com PRM era baixo (Figura 2). Entre os avaliados, 62\% não possuía ensino fundamental completo, $36 \%$ com ensino fundamental e médio e apenas $2 \%$ apre- sentava curso superior completo. A frequência de pacientes sem ensino fundamental foi maior quando comparado a outro estudo, realizado na região sul do Brasil, que revelou frequência de 10,4\% (10). As diferenças regionais podem ter contribuído para a diferença encontrada. A baixa escolaridade associada à carência de informação pode interferir diretamente na capacidade de entendimento do tratamento prescrito e no cumprimento correto da terapia medicamentosa (13), aumentando os riscos de PRM.

No presente estudo também foi demonstrada a frequência de PRM detectados com base na con- 
Figura 4. Distribuição percentual das classes terapêuticas envolvidos em Problemas Relacionados aos Medicamentos (PRM) entre pacientes atendidos no pronto socorro do Hospital das Clínicas Samuel Libânio, Pouso Alegre, MG, Brasil (janeiro-abril de 2019).

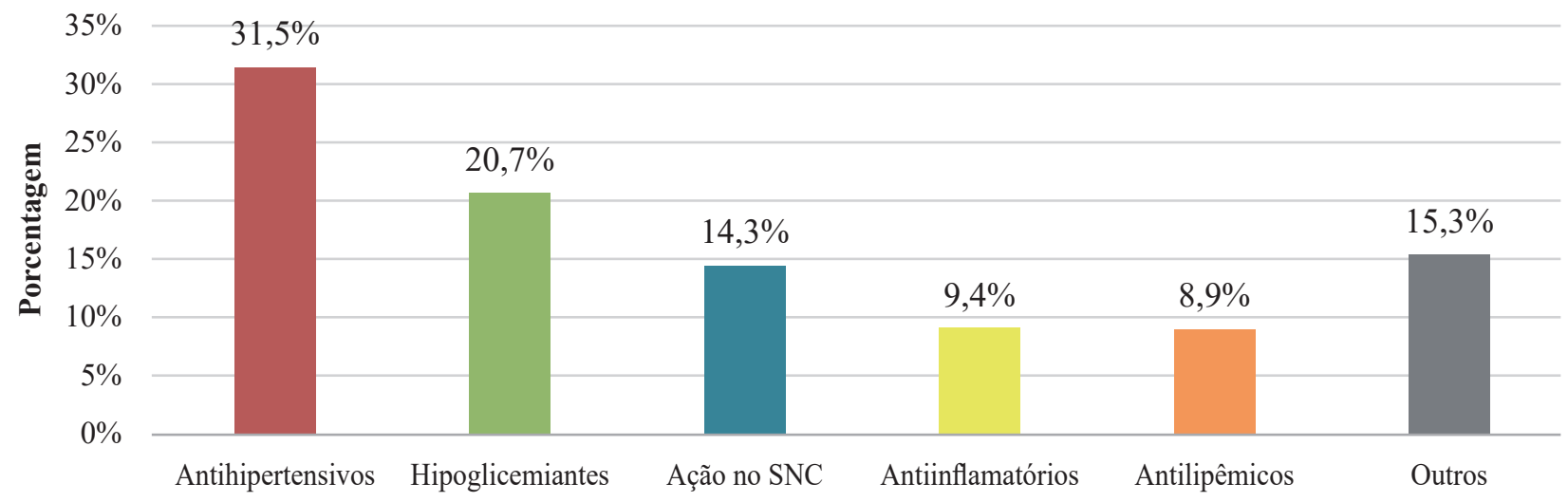

SNC: Sistema Nervoso Central

Figura 5. Distribuição percentual das doenças previamente diagnosticadas e relatadas pelos pacientes atendidos no pronto socorro do Hospital das Clínicas Samuel Libânio, Pouso Alegre, MG, Brasil (janeiro-abril de 2019).

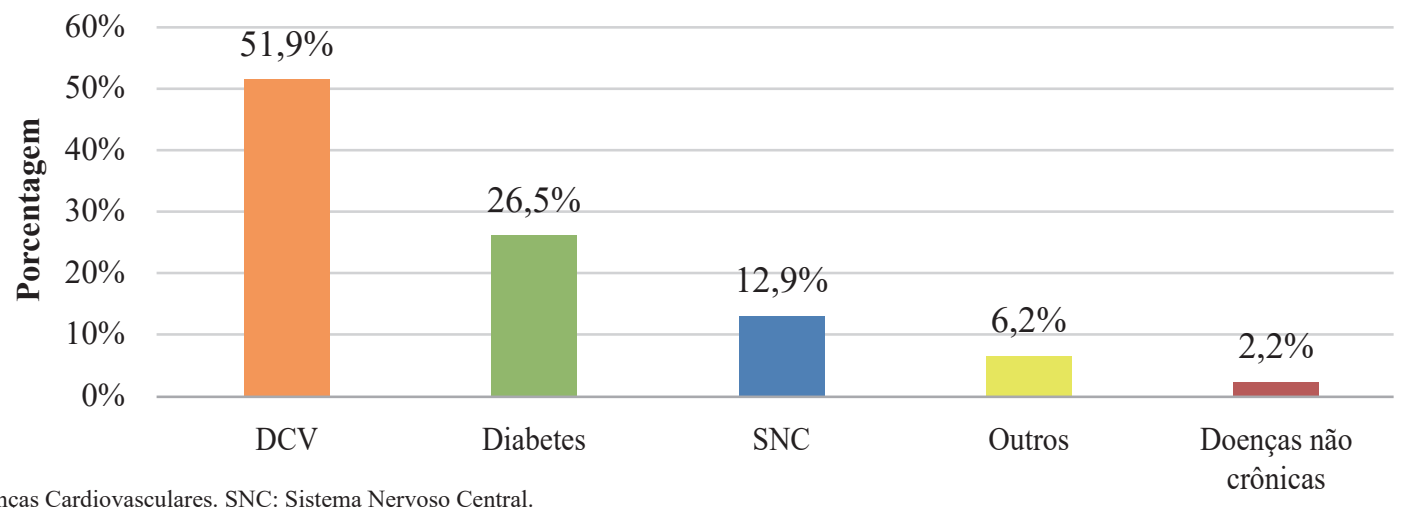

DCV: Doenças Cardiovasculares. SNC: Sistema Nervoso Central.

dição clínica e classificação de risco dos pacientes (sintomas/ queixas/ causas/ parâmetros fisiológicos relatados e apresentados) que procuraram atendimento no pronto socorro do HCSL. Dos pacientes selecionados para o estudo, o maior percentual de pacientes com suspeita de PRM estava em indivíduos classificados com pulseiras amarelas (36\%), que representa nível de gravidade urgente, e laranja $(35 \%)$, que representa nível de gravidade muito urgente (Figura 3).

Foi observado também que a maior parte dos pacientes que apresentaram PRM, de acordo com a avaliação realizada, procurou o OS devido a condições clínicas que os classificaram como urgente ou muito urgente; porém, não pode ser identificado se a apresentação de tais condições clínicas tenha sido devida diretamente ao PRM detectado. Dentre as classes de medicamentos utilizadas, houve alta frequência de uso de medicamentos anti-hipertensivos (31,5\%), seguido de medicamentos hipoglicemiantes $(20,7 \%)$ e de ação no sistema nervoso central (SNC), como antidepressivos, indutores de sono, anticonvulsivantes, entre outros $(14,3 \%)$, o que reflete o perfil clínico dos pacientes, considerando que na amostra houve grande número de pacientes com hipertensão arterial (51,9\%), diabetes mellitus (26,5\%) e com doenças que afetam o SNC (12,9\%), (Figura 4 e 5). Foram observadas diferenças entre 
Figura 6. Distribuição percentual do perfil de adesão ao tratamento farmacológico entre pacientes atendidos no pronto socorro do Hospital das Clínicas Samuel Libânio, Pouso Alegre, MG, Brasil (janeiro-abril de 2019).

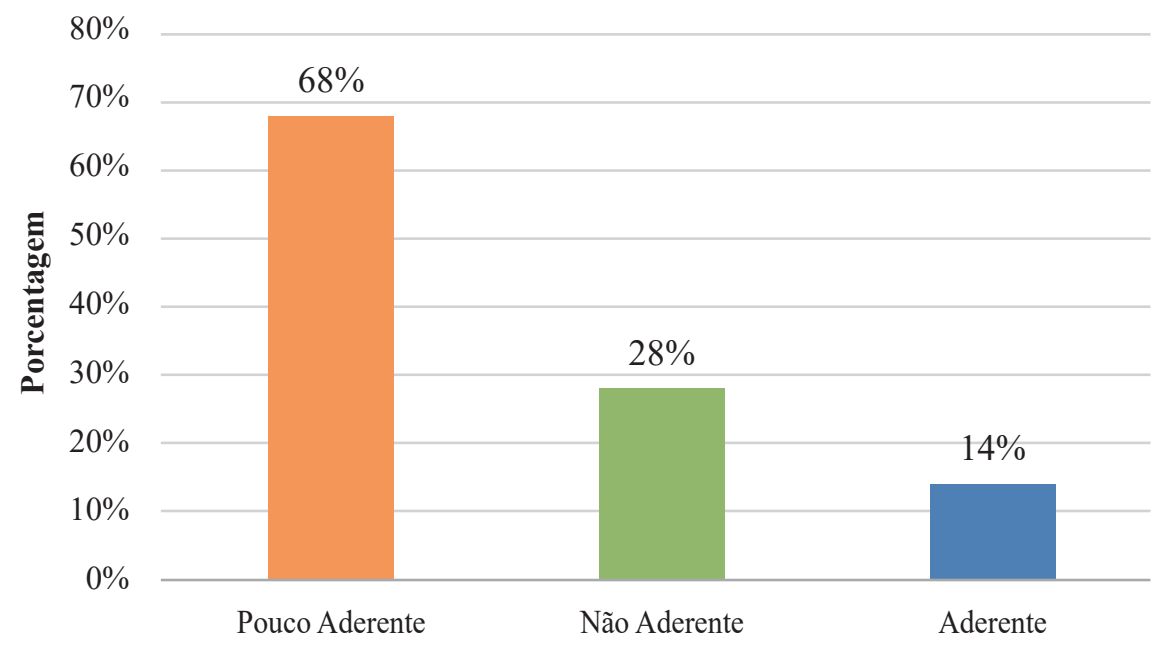

Figura 7. Distribuição percentual dos Problemas Relacionados aos Medicamentos (PRM) observados farmacológico entre pacientes atendidos no pronto socorro do Hospital das Clínicas Samuel Libânio, Pouso Alegre, MG, Brasil (janeiro-abril de 2019).

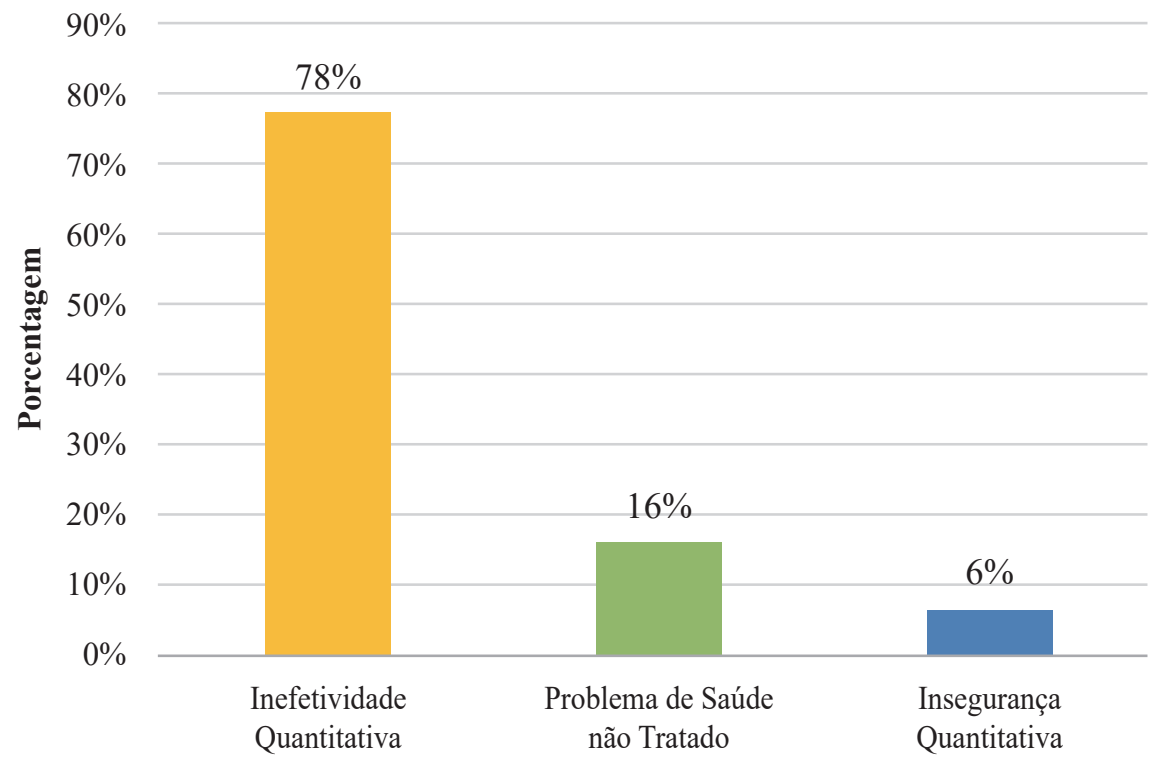

os resultados obtidos no presente estudo comparado a s um estudo realizado no sul do Brasil (12). Essa disparidade pode ser devida às diferenças entre as populações estudadas.

Com relação ao padrão de adesão da população estudada ao tratamento farmacológico, apenas $14 \%$ dos pacientes eram aderentes, $68 \%$ pouco aderentes e $28 \%$ não eram aderentes ao regime terapêutico (Figura 6). Ao investigar as principais causas associadas à baixa adesão ao tratamento farmacológico, foi constatado que $68 \%$ dos pacientes não administravam os medicamentos na dosagem e/ou na frequência de acordo com o descrito na prescrição médica ou esqueciam frequentemente de tomar os medicamentos, apresentando dificuldade em cumprir o regime terapêutico. As principais 
razões apontadas foram a dificuldade na autoadministração dos medicamentos, por não ter alguém na família para auxiliar; dependência de terceiros; polifarmácia (existência de várias administrações diárias); falta de esclarecimento por parte dos profissionais da saúde sobre a importância de se tomar os medicamentos de forma correta para prevenção e/ou controle da doença. Estes dados mostram a ocorrência de erros relacionados a terapia medicamentosa na população desse estudo, o que pode trazer diversos prejuízos para o paciente, ineficácia no tratamento e aparecimento ou complicações de problemas de saúde (13).

Em um estudo realizado no sul do Brasil, por Dall' Agnol e cols. (2004), foram observados problemas de adesão em 43,8\% dos pacientes atendidos no serviço de urgência-emergência do hospital de Nossa Senhora da Conceição, na cidade de Tubarão, Santa Catarina (11). A falta de adesão também foi o motivo mais frequente observado em pacientes admitidos no serviço de emergência com problemas relacionados aos medicamentos, com incidência de 47,2\% (14). Portanto, a baixa adesão ou o não cumprimento no tratamento é considerado o grande desafio do sistema público de saúde, principalmente no enfretamento das condições crônicas $(14,15)$.

Os tipos de PRM encontrados no presente estudo foram: inefetividade quantitativa (78\%), problema de saúde não tratado (16\%) e insegurança quantitativa (6\%). Nos participantes desse estudo, não foi observado qualquer caso de insegurança não quantitativa e inefetividade não quantitativa (Figura 7). Os resultados evidenciaram alta prevalência de PRM de inefetividade quantitativa devido à existência de erros relacionados ao uso incorreto dos medicamentos, identificados na maioria dos pacientes que usavam medicamentos em doses e frequência inferior à prescrição médica afetando a efetividade terapêutica.

Esta ocorrência justifica a incidência de pacientes atendidos no pronto socorro do HCSL para tratar resultados clínicos negativos, complicações e até mesmo surgimento de novos problemas de saúde. Este dado é muito superior a incidência encontrada em outros estudos que mostraram frequência de PRM em torno de 20\% (11).

A não adesão é considerada a principal causa de falha farmacológica especialmente em doenças crônicas (16). Um medicamento é considerado inseguro quando produz ou agrava os problemas de saúde (4). Houve incidência de 6\% de PRM do tipo insegurança quantitativa, mas esse resultado é inferior em relação a incidência de 28,5\% $(4,10)$. Estes pacientes apresentaram suspeitas de RNM por utilizar os medicamentos acima da dose recomendada. A incidência de PRM relacionado ao grupo necessidade, em que o paciente sofre de um problema de saúde em consequência de não receber um medicamento que necessita, foi de $16 \%$. Em outros estudos, essa incidência variou de $2,4 \%$ a $36,4 \%$ $(10,11,17)$.

\section{CONCLUSÃO}

Foi observado que os casos de PRM atendidos no pronto socorro do HCSL eram mais frequentes especialmente em pacientes que possuíam diagnóstico prévio de doenças crônicas e utilizavam medicamentos de forma incorreta, indivíduos com idade acima de 60 anos, não adesão ao tratamento (não cumprimento do regime terapêutico), situação recorrente observada nos pacientes.

Diante desse cenário é necessário que gestores e profissionais de saúde desenvolvam ações centradas no paciente na perspectiva da atenção primária, pautada na prevenção, oferecendo atendimento abrangente que permita a identificação, e resolução de PRM, com objetivo de minimizar os fatores de riscos que podem influenciar negativamente na qualidade de vida dos pacientes. 
1. Luchessi AD; Marçal BF; Araujo GF; Uliana LZ; Rocha MRG; Pinto TJA. Monitoração de propaganda e publicidade de medicamentos: âmbito de São Paulo. Rev Bras Ciênc Farm. 2005;41(3):346-349. DOI: 10.1590/ S1516-93322005000300007

2. Guarido CF; Oliveira ER. Perfil da automedicação de indivíduos residentes na cidade de Marília - SP. Infarma. 2011;23(9/12):39-42.

3. Dall'agnol RSA. Identificação e quantificação dos problemas relacionados com medicamentos em pacientes que buscam atendimento no serviço de emergência do HCPA [Dissertação]. Porto Alegre: Universidade Federal do Rio Grande do Sul; 2003.

4. Hernández DS; Castro MMS; Dáder MJF. Método Dáder: manual de seguimento farmacoterapêutico. 3. ed. Alfenas: Universidade Federal de Alfenas: 2014.

5. Ferrández O; Casañ B; Grau S; Louro J; Salas E; Castells X; Sala M. Análisis de los problemas relacionados con los medicamentos en un hospital de tercer nivel de Barcelona. Gac Sanit. 2019;33(4):361-368. DOI: 10.1016/j.gaceta.2018.01.002.

6. Santos HP; Iglésias F; Limós FL; Faus MJ; Rodrigues LM. Segundo consenso de Granada sobre problemas relacionados con medicamentos. Ars Pharm. 2004; 17:59-66.

7. Carvalho FD. Avaliação econômica do impacto da atividade de atenção Farmacêutica na assistência à saúde: aspectos metodológicos [Dissertação]. Ribeirão Preto: Universidade de São Paulo; 2007.

8. Varallo FR. Internações hospitalares por Reações Adversas a Medicamentos (RAM) em um hospital de ensino [Dissertação]. Araraquara: Universidade Estadual Paulista Júlio de Mesquita Filho; 2010.

9. Cubero-Caballero S; Torres-Murillo JM; CamposPérez MA; Gómez Del Río S; Calleja-Hernández MA. Problemas relacionados com los medicamentos en el area de observación de urgencias de um hospital de tercer nivel. Farm Hosp. 2006;30(3):187-192. DOI: 10.1016/ S1130-6343(06)73970-8.
10. Andreazza RS; Castro MS; Koche PS; Heineck I. Causes of drug-related problems in the emergency room of a hospital in southern Brazil. Gac Sanit. 2011;25(6):501506. DOI: 10.1016/j.gaceta.2011.05.016.

11. Medeiros Netto AS; Melo FB; Silva WB. Frecuencia de problemas relacionados con los medicamentos en pacientes que visitaron el servicio de urgencia de un hospital regional. Seguim Farmacoter. 2005; 3(4):213-224.

12. Dall'Agnol RSA; Albring DV; Castro MS; Heineck; I. Problemas relacionados com medicamentos em serviço de emergência de Hospital Universitário do Sul do Brasil. Estudo Piloto. Acta Farm Bonaer. 2004; 23(4):540-545.

13. Carvalho PT; Okuno MFP; Campanharo CRV; Lopes MCBT; Batista REA. Conhecimento dos pacientes sobre a prescrição medicamentosa no serviço de emergência. Rev Bras Enferm. 2018;71(2):351-357. DOI: 10.1590/0034-7167-2017-0002.

14. Olah YH; Triab KM. Admissions through the emergency department due to drug-related problems. Ann Saudi Med. 2008;28(6):426-429. DOI: 10.5144/0256-4947. 2008.426.

15. Souza TT; Godoy RR; Pontarolo R; Pontarolo R; Fernandez-Llimos F; Correr CJ. Morbidade e mortalidade relacionadas a medicamentos no Brasil: revisão sistemática de estudos observacionais. Rev Ciên Farm Básica Aplic. 2014;35(4):519-532.

16. García-Jiménez E; Amariles P; Machuca M; ParrasMartín M; Espejo-Guerrero J; Faus MJ. Incumplimiento, problemas relacionados con los medicamentos y resultados negativos asociados a la medicación: causas y resultados en el seguimiento farmacoterapéutico. Ars Pharm. 2008;49(2):145-157.

17. Santamaría-Pablos A; Redondo-Figuero C; Baena MI; Faus MJ; Fabric R; Acha O; Novo FJ. Resultados negativos asociados con medicamentos como causa de ingreso hospitalario. Farm Hosp. 2009;33(1):12-25. 